\title{
A numerical investigation of a piezoelectric surface acoustic wave interaction with a one-dimensional channel
}

\author{
S. Rahman ${ }^{1}$ * M. Kataoka ${ }^{1}$, C. H. W. Barnes ${ }^{1}$, and H. P. Langtangen ${ }^{2}$ \\ ${ }^{1}$ Cavendish Laboratory, Madingley Road, Cambridge, CB3 OHE, United Kingdom and \\ ${ }^{2}$ Simula Research Laboratory, Martin Linges v 17, Fornebu P.O.Box 134, 1325 Lysaker, Norway
}

(Dated: May 2, 2017)

\begin{abstract}
We investigate the propagation of a piezoelectric surface acoustic wave (SAW) across a $\mathrm{GaAs} / \mathrm{Al}_{X} \mathrm{Ga}_{1-X}$ As heterostructure surface, on which there is fixed a metallic split-gate. Our method is based on a finite element formulation of the underlying equations of motion, and is performed in three-dimensions fully incorporating the geometry and material composition of the substrate and gates. We demonstrate attenuation of the SAW amplitude as a result of the presence of both mechanical and electrical gates on the surface. We show that the incorporation of a simple model for the screening by the two-dimensional electron gas (2DEG), results in a total electric potential modulation that suggests a mechanism for the capture and release of electrons by the SAW. Our simulations suggest the absence of any significant turbulence in the SAW motion which could hamper the operation of SAW based quantum devices of a more complex geometry.
\end{abstract}

PACS numbers: 85.35.Gv, 73.23.-b, 77.65.Dq

\section{INTRODUCTION}

Surface acoustic waves (SAWs) are widely used in microwave circuit components, such as filters and resonators $\stackrel{1}{\underline{1}}$ In condensed matter physics research, SAWs have been a useful tool in probing electronic structure, for example, in thin metal films,,$\frac{2}{,}$ and quantum Hall liquids, $\stackrel{3}{\underline{3}}$ for a number of decades. In 1996, Shilton et al. realized a device which carries a quantized number of electrons through a quasi-one-dimensional channel (Q1DC) using SAWs. ${ }^{4}$ Such devices are currently being developed for metrological applications and for quantum logic circuits,$\underline{5}$

Despite the enthusiasm for using SAWs in such applications, experimental progress in this direction has been slow. One of the main reasons for this is that we lack a rigorous understanding of the dynamics of the SAW propagation in complicated device structures, and are therefore unable to precisely design or simulate the devices. Earlier analytical work on SAW single-electron transport (SET) devices 6.7 .8 required crude approximations, especially regarding the effect of surface gates, where a realistic two-dimensional model of a split-gate device had not been achieved.

In this paper, we present the results of a numerical investigation of the dynamics at the depth of a two-dimensional electron gas (2DEG) in a $\mathrm{GaAs} / \mathrm{Al}_{X} \mathrm{Ga}_{1-X} \mathrm{As}$ heterostructure, resulting from the propagation of a piezoelectric SAW through a realistic split-gate. The numerical method implemented, ${ }^{9}$ fully takes into account the geometry and material composition of the device, as well as the full two-way coupling between the electrical and mechanical fields. Our simulations are performed in the strongly screened, low SAW power, low barrier height, regime, as this allows us to implement a simple model for the 2DEG and allows assumptions on the SAW amplitude and split-gate barrier height.

We use our solutions to discuss the effects due to the presence of mechanical and electrical surface gates. Although in this paper we restrict our attention to a splitgate device, our method is in principle applicable to a device of any geometry.

This paper proceeds as follows. In Section [II we describe the details of the underlying theory of SAW propagation in a gated device. We discuss the earlier work of Aizin and Gumbs 6.7 pointing out the differences between our formalisms and solutions. In Section IIII we describe the method behind our numerical strategy. The results for an un-gated device and a split-gate device are presented in Sections IVA and IVB respectively.

\section{SINGLE-ELECTRON SAW DEVICES}

The quantization of acoustoelectric current was first

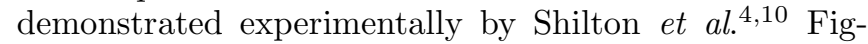
ure凹(a) shows the experimental setup used. It consists of a GaAs $/ \mathrm{Al}_{X} \mathrm{Ga}_{1-X}$ As heterostructure containing a twodimensional electron gas (2DEG) that is formed into a mesa by wet etching. A Q1DC is formed when a negative potential is applied to a split metallic surface gate. A large negative voltage applied to the gates induces a narrow depleted constriction between the two 2DEG regions. Application of a microwave signal to an interdigitated transducer excites electromechanical waves through the piezoelectric effect which include both SAWs and bulk waves.

The electric component of the SAW captures electrons from the 2DEG in its potential minima transporting them through the constriction produced by the split-gate potential. The current measured from the drain region exhibits a step-like behavior as shown in Fig. T(b). The values of the current plateaus are quantised with

$$
I=n e f
$$

where $I$ is the current, $e$ is the electron charge, $n$ is the 
(a)

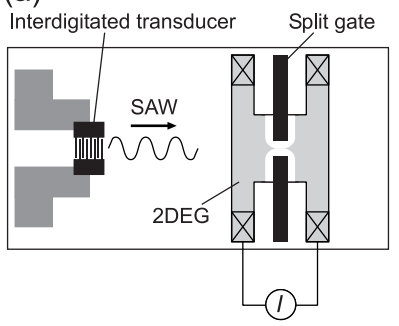

(b)

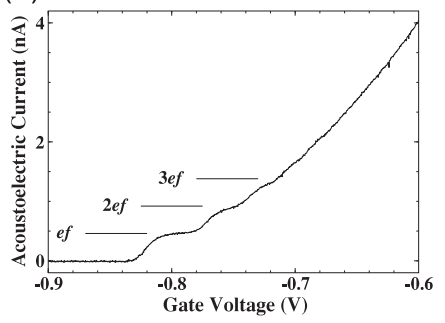

FIG. 1: (a) A schematic diagram showing the experimental setup in acoustic charge transport experiments. A transducer on the left excites a SAW wave that propagates towards the spit-gate on the right. (b) The acoustoelectric current versus split-gate voltage. The current exhibits a step-like behavior as the gate voltage is varied.

number of electrons transported, and $f$ is the SAW frequency. The case with $n=1$ involves the transport of a single electron per cycle through the Q1DC.

The original explanation for the quantization of acoustoelectric current given by Shilton et al. ${ }^{4.10}$ asserts that the combination of the SAW electrostatic potential and the split-gate potential produces a travelling quantum dot that transports a fixed number of electrons from one side of the constriction to the other. This is plausible as the length of the depleted region formed by the splitgate $(\sim 1.5 \mu \mathrm{m})$ is a small fraction greater than SAW wavelength $(\sim 1 \mu \mathrm{m})$. Several electrons are believed to be initially trapped in each SAW minimum but as it passes through the constriction formed by the split-gate, its physical dimensions decrease and Coulomb repulsion between electrons forces electrons out, thus determining the final number of electrons remaining in the dot. This explanation, although qualitatively satisfactory, was not at the time, supported by a detailed theoretical analysis involving an accurate model for the SAW and split-gate, which we aim to develop in this paper.

One approach for such an analysis, is to solve the equations of motion in the GaAs $/ \mathrm{Al}_{X} \mathrm{Ga}_{1-X}$ As media, taking into account the complex geometry of the split-gate, the material composition and external electric fields, to determine the evolution of the SAW potential through the device. The resulting electric potential could be used as a part of a quantum-mechanical treatment to describe the evolution of the electronic states.

However, this approach is a non-trivial one, as the equations of motion in a piezoelectric material such as $\mathrm{Al}_{X} \mathrm{Ga}_{1-X} \mathrm{As}$, consist of a set of four coupled partial differential equations, second order in time and space. Moreover, as the SAW passes through the split-gate potential, initially $\sim 30$ electrons are captured from the 2DEG, this number reduces as the SAW quantum dot is dragged into the increasing electric field in the constriction. This problem then involves a solution to the time-dependent many-particle Schrödinger equation including spin. To perform this calculation exactly is beyond the scope or intention of this paper. The focus of

this paper is the solution of the equations of motion in the GaAs $/ \mathrm{Al}_{X} \mathrm{Ga}_{1-X}$ As medium and, using a simple model for the 2DEG and, ignoring any self consistent effects, the calculation of the SAW potential as it traverses the split-gate. The equations of motion in a heterogeneous piezoelectric material are

$$
\begin{gathered}
\varrho \frac{\partial^{2} u_{i}}{\partial t^{2}}=\frac{\partial}{\partial x_{j}} c_{i j k l}^{E} \frac{\partial u_{l}}{\partial x_{k}}+\frac{\partial}{\partial x_{j}} e_{k i j} \frac{\partial \phi}{\partial x_{k}} \\
\frac{\partial}{\partial x_{i}} \epsilon_{i j}^{S} \frac{\partial \phi}{\partial x_{j}}=\frac{\partial}{\partial x_{i}} e_{i j k} \frac{\partial u_{k}}{\partial x_{j}}
\end{gathered}
$$

where $u_{i}$ is a three-component vector representing the displacement of the material at each point, $\phi$ is the electric potential, $c_{i j k l}^{E}$ are components of the elastic tensor, $\epsilon_{i j}^{S}\left(=\epsilon^{S}\right)$ are components of the material dielectric (the superscripts $E$ and $S$ indicate that the values were determined under constant electric field and strain, respectively), $\varrho$ is the mass density and $e_{i j k}$ are components of the piezoelectric tensor representing the coupling strength between the electric and mechanical fields. The Eqs. (2) can be regarded as a system of wave equations with a load term due to the electric field, and Eq. (3) can be regarded as a Poisson equation with a source term due to the mechanical deformations.

The equations are solved with traction-free boundary conditions. For example $\sigma_{i z}=0$ for the surface normal to the $z$ axis, where $\sigma_{i j}$ are components of the stress tensor, defined by

$$
\sigma_{i j}=-e_{k i j} E_{k}+c_{i j k l}^{E} \varepsilon_{k l},
$$

where $E_{k}=-\frac{\partial \phi}{\partial x_{k}}$ and $\varepsilon_{i j}=\frac{1}{2}\left(\frac{\partial u_{i}}{\partial x_{j}}+\frac{\partial u_{j}}{\partial x_{i}}\right)$. The electrostatic boundary conditions require the prescription of the normal component of the electric displacement at the free surface of the medium. The electric displacement vector is defined by

$$
D_{i}=\epsilon_{i j}^{S} E_{j}+e_{i j k} \varepsilon_{j k} .
$$

Aizin et $a l \underline{\underline{6}}$ followed this approach providing a closed form analytic formula for the combined potential of the SAW and split-gate. The calculation involved the simplification of Eqs. (2) and (3), and of the description of the physical system, so that analytical solutions were tractable. In particular, their approach was twodimensional, taking into account the direction of SAW propagation and the direction into the bulk of the heterostructure only. Clearly, this analysis cannot take into account the geometry of the surface gates and therefore the 'split' nature of the split-gate. In fact, a split-gate was approximated to be a single bar gate. Another simplification was the decoupling of the mechanical motion from the electric fields by setting the piezoelectric term in 
Eq. (2) to zero. This simplification was based on the fact that $e_{i j k} \ll c_{i j k l}$ for $\mathrm{Al}_{X} \mathrm{Ga}_{1-X}$ As. In practice, however, externally applied voltages (and hence electric fields) may be large enough that they contribute to the mechanical strains and hence the observed potential in the vicinity of the gates ${ }^{9}$ Thus, the term with $e_{i j k}$ in Eq. (2) cannot always be neglected. Their calculations also ignore differences in the elastic constants and mass density between the gates and the heterostructure substrate. The gates are often fabricated from a combination of aluminium $(\mathrm{Al})$, gold $(\mathrm{Ag})$ or titanium $(\mathrm{Ti})$. In fact, this approach neglects the mechanical presence of the gates at all: the SAW potential $\phi_{S A W}$ is effectively assumed to have a cosine form i.e. $\phi_{S A W} \sim \cos (k x-\omega t)$ and is added to the split-gate potential $\phi_{S G}$ which is of a quadratic form, to give the total electric potential $\phi$. In practice however, the mechanical presence of surface gates could cause the SAW potential to be damped, reflected or diffracted in the vicinity of the gates. The presence of surface gate screening has been utilized in SAW-based photoluminescence experiments 12.13

However, using the potential $\phi$, Aizin and Gumbs were able to show that if tunnelling of electrons from the quantum dot to the source 2DEG was allowed, they could explain the transition in the acoustoelectric current from the point where no charge is transported, to that where a single electron is transported ${ }^{\underline{6}}$ In effect, they provided an explanation of the first current plateau. In subsequent theoretical work, $\frac{8}{\underline{B}}$ Gumbs et al. showed, using the same potential, that the second quantized plateau in the current as a function of the gate voltage or SAW power can be explained by the effect of both the Coulomb blockade in the quantum dot and the backward tunnelling into the Q1DC.

The analytic approach by Aizin and Gumbs was a convenient one, and similar approaches for the SAW and gate potential have been adopted in other theoretical works. $14,15,16$

Our recent numerical investigation, 9 based on the finite element formulation of Eqs. (21) and (31) demonstrated both the effect of the mechanical presence of a gate on the SAW electric potential and the presence of a large static electric potential on the mechanical strains in the material. The procedure includes the full two-way coupling between the electric and mechanical fields. The advantage of the approach is that we can handle the geometry and material composition of the split-gate, or any shape of gate pattern, easily. In particular, the SAW wavefront was shown to be damped and scattered somewhat, after propagating through a single gate. Such effects could prevent the proper functioning of SAW devices, operating in the quantum regime. However, the intention of that paper was to demonstrate that the numerical method is capable of reproducing the fully coupled elasto-electric dynamics of the physical system. In order to exaggerate the effects of the electrical and mechanical coupling, the investigation used fictitious materials for the gate, which had substantially different physical properties to
$\mathrm{Al}$, Ti or Ag, which are frequently used in SAW based SET experiments.

In this paper, we apply our numerical solution strategy to a split-gate device with realistic parameters for the gate geometry and material composition. We also include a simple model for the 2DEG, when a voltage is applied to the split-gate.

\section{METHOD}

Figure 2 illustrates the geometry of the device to be modelled. The device consists of a $\mathrm{GaAs} / \mathrm{Al}_{X} \mathrm{Ga}_{1-X} \mathrm{As}$ $(X=0.3)$ heterostructure with a $\mathrm{Ti} / \mathrm{Al}$ split-gate placed on the surface. The sample has dimensions $12 \mu \mathrm{m} \times$ $3 \mu \mathrm{m} \times 8 \mu \mathrm{m}$ in the $x, y$ and $z$ directions, respectively, with $z=z_{0}\left(z_{0}=8 \mu \mathrm{m}\right)$ at the top surface and the heterostructure occupying the region shown in Fig. 2. From the top surface down, there is a $10 \mathrm{~nm}$ GaAs capped layer, an $80 \mathrm{~nm} \mathrm{Al}_{X} \mathrm{Ga}_{1-X}$ As layer, and the remainder being GaAs. We are interested in calculating the dynamic effects of the SAW propagating through the constriction and therefore we ignore static charge distributions due to doping in the $\mathrm{Al}_{X} \mathrm{Ga}_{1-X}$ As layer. The thickness (or height) of the $\mathrm{Ti}$ and $\mathrm{Al}$ components of the gates are chosen to be $20 \mathrm{~nm}$ and $40 \mathrm{~nm}$, respectively. The constriction formed by the split-gate has dimensions $0.7 \mu \mathrm{m}$ and $1.0 \mu \mathrm{m}$ in the $x$ and $y$ directions, respectively. The gate is centered along the $x$ axis at $x=x_{g}=9850 \mathrm{~nm}$.

To obtain an accurate description of the potential landscape in the device (without a SAW) and in particular at the 2DEG, a three-dimensional Hartree or density functional calculation would be necessary $\frac{17}{1}$ incorporating suitable boundary conditions for the split-gate and charge from donor impurities. Moreover, to incorporate the time-varying polarization charge induced from the SAW, this calculation would need to be performed at every time-step. Such a task would require enormous computational resources. Instead, a simple model for the split-gate induced potential landscape is implemented here, based on our experimental observations. In the experiments, the split-gate potentials are applied relative to the 2DEG. The 2DEG is believed to be depleted a few hundred nano-meters laterally away from the split-gate. Hence, in this model, when a gate voltage is applied, the 2DEG is assumed to be depleted $300 \mathrm{~nm}$ laterally from the split-gate, and is modelled as a metal sheet, with a potential difference applied between the split-gate and the metal sheet (with the potential at the 2DEG set to $0 \mathrm{~V}$ ), in the numerical solution of the Eq. (2). For the electrical boundary conditions at the free surface, we choose a Neumann-type condition on the un-gated regions, implemented by setting $D_{z}=0$ in order that overall charge neutrality in the device is satisfied ${ }^{18,19}$ Dirichlet-type conditions are unsuitable for the un-gated regions as they would compromise the SAW potential. The mechanical boundary conditions implemented are the 'traction-free' conditions defined earlier. 


\begin{tabular}{|c|c|c|c|c|c|c|}
\hline Material & $\begin{array}{c}c_{11} \\
\left(10^{10} \mathrm{Nm}^{-2}\right)\end{array}$ & $\begin{array}{c}c_{12} \\
\left(10^{10} \mathrm{Nm}^{-2}\right)\end{array}$ & $\begin{array}{c}c_{44} \\
\left(10^{10} \mathrm{Nm}^{-2}\right)\end{array}$ & $\begin{array}{c}e_{14} \\
\left(\mathrm{Cm}^{-2}\right)\end{array}$ & $\begin{array}{c}\epsilon \\
\left(\mathrm{Fm}^{-1}\right)\end{array}$ & $\begin{array}{c}\rho \\
\left(10^{3} \mathrm{Kgm}^{-3}\right)\end{array}$ \\
\hline $\mathrm{Al}_{X} \mathrm{Ga}_{1-X} \mathrm{As}$ & $11.88+0.14 X$ & $5.38+0.32 X$ & $5.94-0.05 X$ & $-0.16-0.065 \mathrm{X}$ & $(13.18-3.12 \mathrm{X}) \epsilon_{0}$ & $5.36-1.6 \mathrm{X}$ \\
Titanium & 20.30 & 11.47 & 4.416 & $\mathrm{n} / \mathrm{a}$ & $\mathrm{n} / \mathrm{a}$ & 4.540 \\
Aluminium & 11.09 & 5.842 & 2.626 & $\mathrm{n} / \mathrm{a}$ & $\mathrm{n} / \mathrm{a}$ & 2.698 \\
\hline
\end{tabular}

TABLE I: Physical properties of $\mathrm{Al}_{X} \mathrm{Ga}_{1-X} \mathrm{As}, \stackrel{23}{,} \mathrm{Al}^{24}$ and $\mathrm{Ti}^{24}$. The permittivity of vacuum is $\epsilon_{0}$.

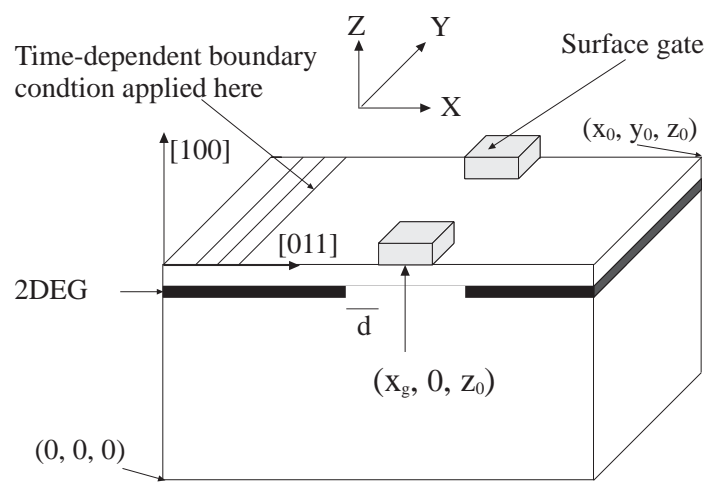

FIG. 2: A schematic diagram showing the device geometry. A split-gate, composed of a $\mathrm{Ti} / \mathrm{Al}$ alloy, is placed on the surface $(z=z 0)$ of the $\mathrm{GaAs} / \mathrm{Al}_{X} \mathrm{Ga}_{1-X}$ As substrate. The SAW, generated by the three membranes separated $1.0 \mu \mathrm{m}$ apart, propagates along the $x$-axis. The $x_{0}, y_{0}$ and $z_{0}$ parameters are chosen to be $12000 \mathrm{~nm}, 3000 \mathrm{~nm}$ and $8000 \mathrm{~nm}$ respectively. The depletion parameter $d$ is $300 \mathrm{~nm}$. The gate is centered along the $x$ axis at $x=x_{g}=9850 \mathrm{~nm}$.

In order to excite the SAWs, we apply a timedependent boundary condition of the form

$$
u_{z}=A \sin (2 \pi f t), \quad x=\text { const }, \quad z=z_{0},
$$

where $f$ is the frequency and $A$ is the amplitude. Here, $f$ is chosen to be $2.7 \mathrm{GHz}$ in order to satisfy the relation $v_{S A W}=f \lambda$, where $v_{S A W}$ is the SAW velocity $\left(\sim 2.7 \times 10^{3} \mathrm{~ms}^{-1}\right)$ and $\lambda$ is the period of the transducer fingers. The amplitude $A$ is chosen such that the SAW has the amplitude of $\sim 20 \mathrm{mV}$ at a depth of $100 \mathrm{~nm}$, and corresponds to the low SAW power regime of the experiments. The oscillation is applied at three values of $x$ separated by $1.0 \mu \mathrm{m}$ as shown in Fig. 2 to increase the SAW amplitude in the $x$ direction relative to $z$ direction: the SAW wave-fronts generated by each membrane add more constructively along the $x$-axis than the $z$-axis.

For $\mathrm{Al}_{X} \mathrm{Ga}_{1-X} \mathrm{As}$, the only non-zero components of the piezoelectric tensor have the value $e_{14}$. Also, the non-vanishing components of the permittivity tensor are $\epsilon_{11}=\epsilon_{22}=\epsilon_{33}=\epsilon_{s}$, and the non-vanishing components of the elastic tensor (not written out above) are $c_{x x x x}=$ $c_{y y y y}=c_{z z z z}=c_{11}, c_{x x y y}=c_{y y z z}=c_{z z x x}=c_{12}$, and $c_{x y x y}=c_{y z y z}=c_{z x z x}=c_{44}$. All other non-zero components of the elastic tensor can be determined by applying its symmetry properties $c_{i j k l}=c_{j i k l}=c_{i j l k}=c_{k l i j}$. The values of these constants for the relevant materials are given in Table I. The dielectric constant and $e_{14}$ are not required for the $\mathrm{Ti}$ and $\mathrm{Al}$ as they are subject to Dirichlet boundary conditions on all external surfaces. In our simulations, we follow the convention in SAW based SET experiments where the crystal orientation is such that the $x$ direction is aligned to [011] direction, and $z$ direction to [100] direction ${ }^{20}$

The finite element method was chosen for its proven ability in handling geometrically complicated domains ${ }^{21.22}$ The basic idea of the finite element method is to approximate the unknown fields, for example $\phi$ in the Poisson equation above, by a linear combination of basis functions $N_{i}, \phi \approx \hat{\phi}=\sum_{j=1}^{n} N_{j} \phi_{j}$, then insert $\hat{\phi}$ in the Poisson equation, and demand the residual to be orthogonal to the space spanned by $\left\{N_{1}, \ldots, N_{n}\right\}$. We utilize finite elements in the spatial part of the problem and use a second order finite difference discretisation in time for computational speed so Eq. (2) is approximated by

$$
\varrho \frac{u^{\ell-1}-2 u^{\ell}+u^{\ell+1}}{\Delta t^{2}}=\operatorname{RHS}^{\ell}
$$

where RHS $^{l}$ is a finite element approximation of the elastic stress term and the mechanical field load. The superscript $\ell$ represents the time level. We use eight-noded brick elements corresponding to linear basis functions $N_{i}$, resulting in an overall spatial and temporal convergence rate for the error, of 2.0. An operator splitting strategy is employed to split the coupled $u_{i}-\phi$ problem ${ }^{22}$ Inheritance and polymorphism principles from object-oriented programming $\stackrel{22}{2}$ are used to incorporate software components from the Diffpack library, for solving the Poisson and the elasticity equations, respectively, thus maximizing efficiency in the programming and verification. The numerical formulation and verification is described in more detail in Ref. 9

\section{RESULTS AND DISCUSSION}

\section{A. SAW through an un-gated surface}

We first demonstrate that our method for exciting acoustoelectric vibrations using three vertically oscillating membranes, excites the SAW modes with the required wavelengths and velocity, by performing a simulation without any surface gates and without the 2DEG. Therefore, it is not necessary to set the total electric potential $\phi$ to $0 \mathrm{~V}$ at the 2DEG in this simulation. (Also, 
the absence of depletion would result in the SAW being invisible everywhere at that depth.) Figure 3 shows the time development of the electric potential as a function of distance along the propagation direction $(+x$ direction). The curves were extracted at a depth of $100 \mathrm{~nm}$ (where the interesting physics of acoustoelectric charge transport take place). The time-dependent boundary condition given by Eq. (6) is applied on the left-hand-side of Fig. 3 with the closest membrane at $x=6600 \mathrm{~nm}$ (not shown). We see that two transient peaks, clearly distinguishable from all the other peaks, are initially formed, and are followed by a more consistent set of peaks which also propagate from left to right of the plots. The transient peaks, which are a common feature of these numerical simulations, are a consequence of the transition from a flat lattice i.e. zero displacement everywhere at $(t=0)$ to one with SAWs $(t>0)$. Moreover, as three membranes are used to generate the SAW, it takes three periods of the SAW to establish a consistent set of peaks. In experiments the transients occur too, but many thousands of SAW wavefronts pass through the split-gate, so the initial transient peaks are actually insignificant. In the following numerical experiments, the transients are included in the analysis as they provide some insight of differing SAW amplitudes, in a single simulation run.

From Fig. 3 we see that in this particular run, the largest amplitude is $\sim 20 \mathrm{mV}$ and the SAW resembles the plane wave shape with the expected velocity $2770 \pm 20 \mathrm{~ms}^{-1}$, and a wavelength of $1.0 \mu \mathrm{m}$. In our previous numerical investigations,,$\frac{9}{}$ we showed that the solution method exhibited the characteristic decay into the bulk as well as a phase difference between the lateral and vertical displacements $u_{x}$ and $u_{z}$, respectively ${ }^{25}$ From Fig. 3, we see that despite the discontinuities in the material parameters due to the the presence of the heterostructure, we are able to produce coherent waves. This is consistent with the fact that the electrical and mechanical properties of $\mathrm{Al}_{X} \mathrm{Ga}_{1-X}$ As as shown in Table I, differ by less than $1 \%$.

\section{B. SAW through a split-gate}

The mechanical effects of the gates are investigated first, by performing a simulation of a SAW through a split-gate and without applying any gate voltages. Dirichlet boundary conditions are therefore not applied to the gates in this case. Moreover, since here we are interested only in the effect of the purely mechanical presence of the gates, we can assume that they have dielectric properties identical to that of the substrate below it. This allows us to avoid divergences in the solution of Eq. (3) due to absence of the dielectric parameter $\epsilon_{i j}^{S}$, for the metals. Figures 4 (a) and 4 (b) compare the SAW potential $\phi$ with and without the mechanical surface gates, directly below the surface gate and at the center of the constriction, respectively. Directly below the gate, the SAW amplitude is seen to be reduced by $\sim 30 \%$, al-

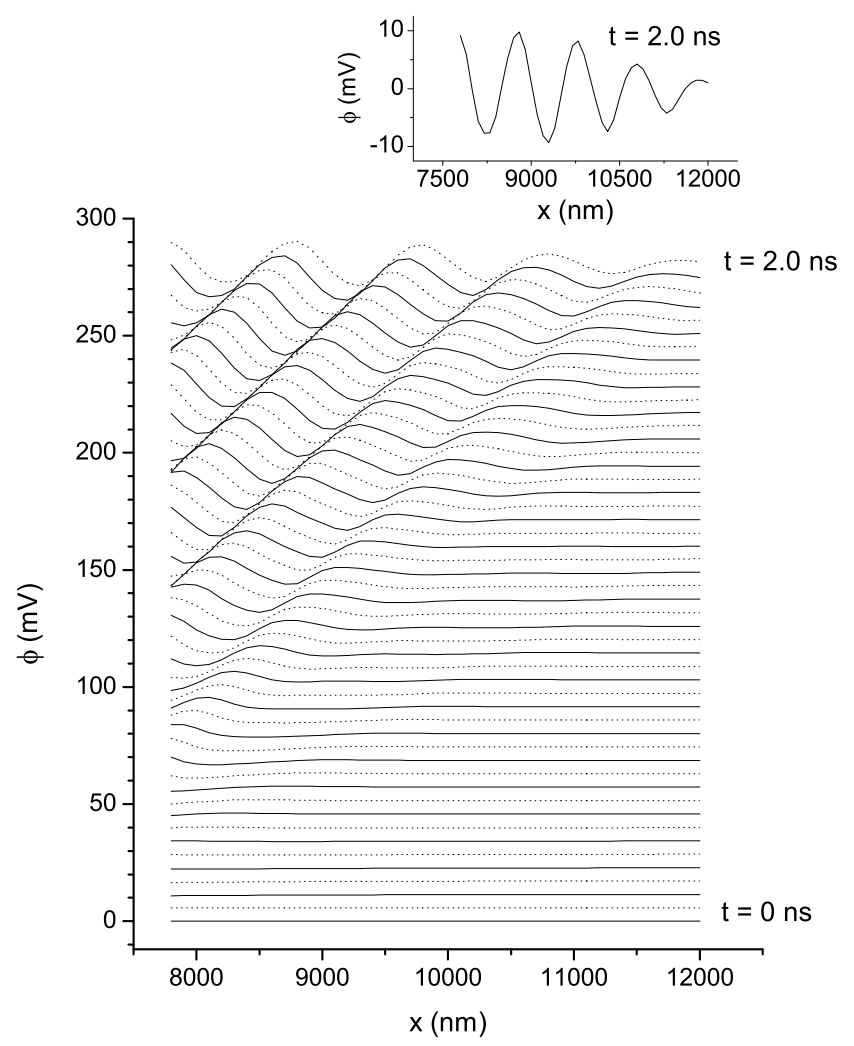

FIG. 3: Time development of SAW induced electric potential $\phi$ as a function of the $x$-axis (without gates on the surface and without the 2DEG). For clarity, the inset shows the amplitude of the SAW is $\sim 20 \mathrm{mV}$.

though the amplitude recovers up to at least $\sim 90 \%$ of its original value after traversing the gate. At the center of the constriction, the difference of amplitudes between the gated and un-gated devices is less than one per-cent in these simulations. The results of Fig. 4 are consistent with the fact that electromechanical energy carried by the SAW is much greater than that could be stored by the gates; the size of the SAW into the depth is over one micron while the height of the gate is typically $\sim 0.05 \mu \mathrm{m}$.

Having established the mechanical effects of the $\mathrm{Ti} / \mathrm{Al}$ split-gates, we now apply an electric potential to the surface gates such that the barrier height due to the splitgate, after ignoring static charges on the surface and donor levels, is $\sim 20 \mathrm{mV}$ at $100 \mathrm{~nm}$ below the surface, corresponding to the low barrier height and short constriction, regime in experiments. From the initial timelevel of the simulation at $t=0$ to the final at $t=2.5 \mathrm{~ns}$, several SAW wavelengths pass through the depleted region. We observe a pattern from the total SAW and gate potential $\phi$, resembling that of an 'electron pump', 26.27 which is repeated with the period of the SAW. Figure 5 actually includes three cycles of this modulation. For the third modulation starting at $t=2.24 \mathrm{~ns}$, a local minimum extends between the source 2DEG and the depleted region. We would expect electrons from the source 2DEG to relax into the minimum. As $t$ increases, a SAW 


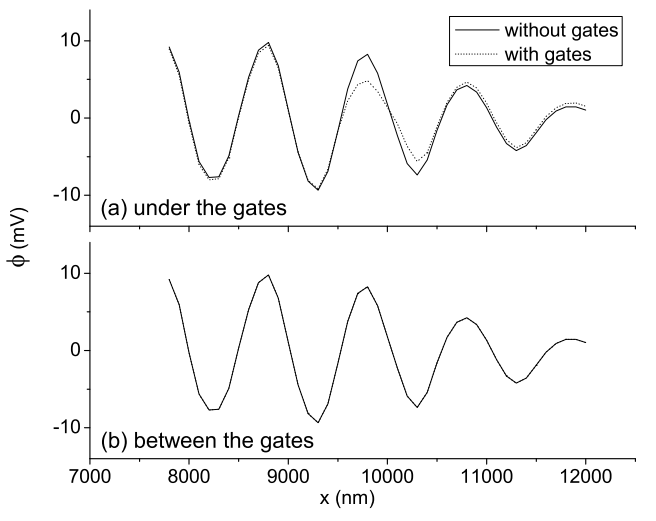

FIG. 4: The SAW potential $\phi$ at $t=2.0 \mathrm{~ns}$, (a) directly under the gates $(y=500 \mathrm{~nm})(\mathrm{b})$ in between the gates at the center of the split-gate constriction $(y=1500 \mathrm{~nm})$. The curves were taken $100 \mathrm{~nm}$ below the surface. The SAW amplitude is reduced directly below the gate as much as $\sim 30 \%$ but less than $\sim 1 \%$ at the center of the constriction.

maximum enters the depleted region forming a potential barrier against electrons escaping or tunnelling backward into the source 2DEG while the SAW maximum in front acts against escaping or tunnelling forward into the drain 2DEG. The electrons are thus confined in the SAW minimum and are transported along with it, over the potential barrier formed by the split-gate, until the forward SAW maximum leaves the depleted region and becomes screened by the drain 2DEG.

Figure 5 suggests that the first two pump motions, which are caused by the transients in the SAW and thus have a smaller amplitude, have a lower probability to transport electrons through the channel, as the potential minima that extend from the source 2DEG to the depleted region are smaller, producing overall smaller minima when combined with the gate potential. Therefore, fewer states will be available in the dot for the electrons to occupy. For these transients, the rear tunnel barrier are also smaller and therefore electrons have a higher probability to escape to the source 2DEG. This is consistent with Gumbs' investigation, ${ }^{7}$ which showed the acoustoelectric current increase as the ratio of the SAW potential amplitude to the height of the gate induced barrier, is increased, despite a different reasoning based on analytic models for the SAW and split-gate potentials.

Experiments have been performed in low SAW power and low barrier height regime, which utilized SAWs in conjunction which a quantum dot to effect a pumping modulation of the total electric potential ${ }^{28}$

Figure 6] shows the curve plots of the total electric potential $\phi$ for times $t$ between $2.24 \mathrm{~ns}$ and $2.34 \mathrm{~ns}$ when a SAW maximum enters the depleted region. It is clear that the rear potential barrier becomes wider and taller with time, thus reducing the probability of electrons escaping or tunnelling backwards into the 2DEG. These results suggest that the number of electrons transported through the dot is determined early on in the capture

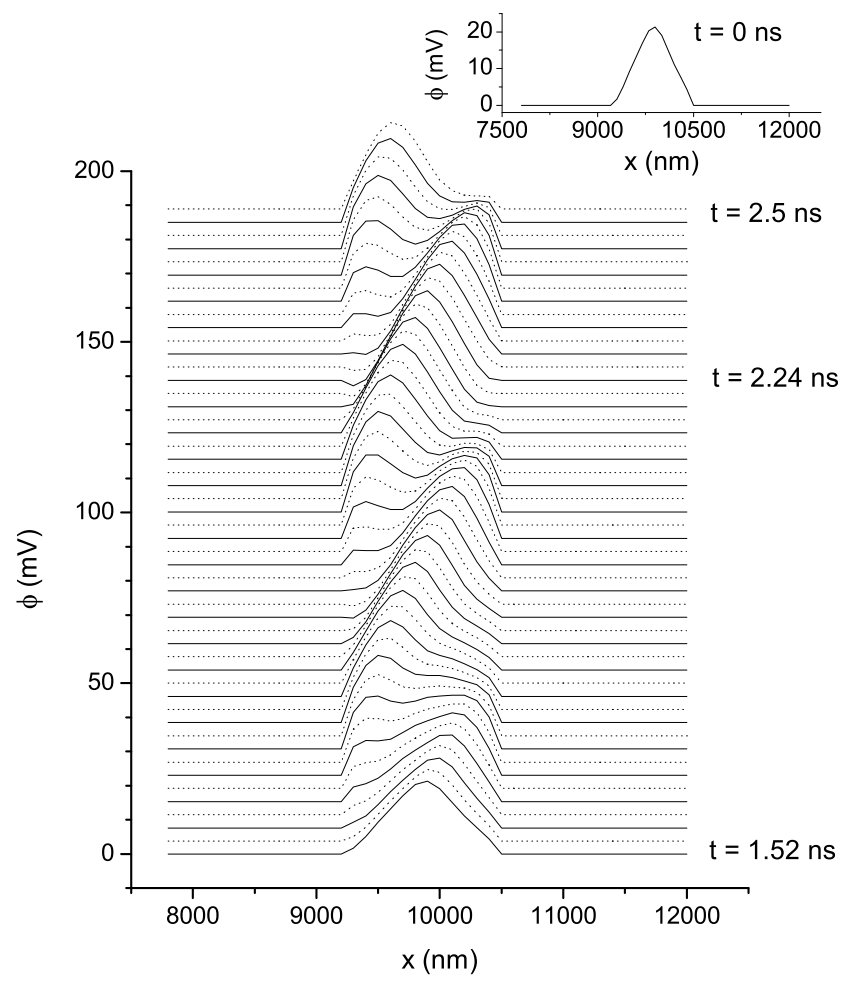

FIG. 5: Time development of SAW induced electric potential $\phi$ as a function of the $x$ axis. The simulation includes the screening by the 2DEG, in the source and drain regions.

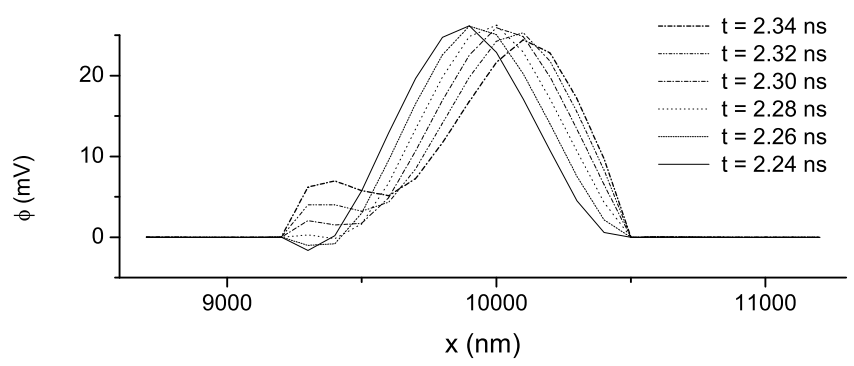

FIG. 6: The time development of a rear potential barrier showing the mechanism by which electrons from the source 2DEG could be captured.

process.

In much of the previous theoretical work, $\stackrel{6.7 .8}{t}$ the increase in width and height of the rear potential barrier has not formed a significant part of the analysis. However, Flensberg et al.16 demonstrated that the rapid change of the SAW potential barrier at the entrance to the channel results in a rapid reduction of tunnelcoupling between the source 2DEG and the SAW minimum inducing non-adiabaticity in the travelling dot. Flensberg et al. then showed that the non-adiabaticity sets a limit on the accuracy of the quantization plateaus.

In the model of Robinson and Barnes 14 the tunnel barrier decreases with time, and the number of electrons in the dot is determined when the SAW minima reach the 


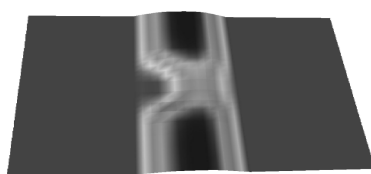

(a) $t=2.300 \mathrm{~ns}$

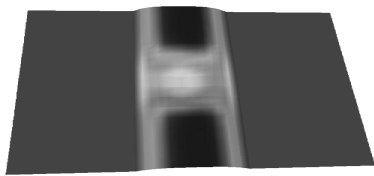

(c) $t=2.375 \mathrm{~ns}$

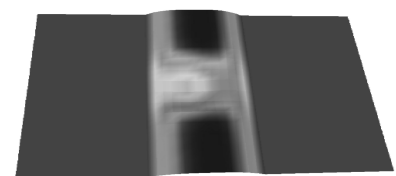

(b) $t=2.350 \mathrm{~ns}$

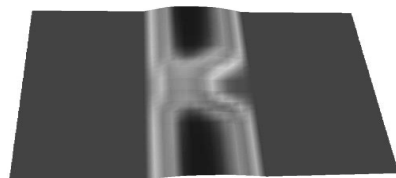

(d) $t=2.500 \mathrm{~ns}$
FIG. 7: Surface plots showing the time development of the SAW induced electric potential $\phi$ in the $x-y$ plane.

point of maximum gradient of the split-gate potential barrier. However, their work was based in the high SAW power and high barrier height, regime and cosine and gaussian models for the SAW and split-gate, respectively, were implemented.

Figure 7 shows surface plots of the electric potential $\phi$ on a two-dimensional surface on the $x-y$ plane, $100 \mathrm{~nm}$ below the surface at a sequence of times $t$ providing a more vivid illustration of the pumping mechanism. In particular, at $t=2.35 \mathrm{~ns}$ a quantum well begins to form on the left hand side of the split-gate as a SAW minimum enters the constriction. At $t=2.375 \mathrm{~ns}$ the SAW minimum is located in between the gates, forming a well defined quantum well of a circular geometry (although the geometry will vary from circular to elliptical depending on the split-gate potential). At $t=2.5 \mathrm{~ns}$, the SAW minimum exits the constriction, and the well begins to disappear.

Figures 8 (a) and 8 (b) show curve-plots of the total electric potential $\phi$ parallel to the $y$-axis, through the center of the constriction. From Fig. 8 (a), one can see the oscillations of the total electric potential $\phi$ as the SAW propagates through it. The amplitude of the oscillations at the center of the constriction is $\sim 20 \mathrm{mV}$, about the same amplitude as that of the bare SAW (i.e., without gates), whereas the SAW amplitude below the gate is $\sim 5 \mathrm{mV}$ and therefore has been reduced by $\sim 75 \%$. This is due to the screening of the SAW potential by the gates, the presence of mechanical gates as discussed earlier, and to a much lesser extent, due to the mechanical strains caused by the gate voltage affecting the SAW motion ${ }^{9} \cdot 31$ These figures also show the a change in the SAW frequency under the gates, in the form of 'splitting' of the peaks, where labelled. In particular, the SAW oscillates at a higher frequency under gates.

The peak splitting or increase in the frequency of the SAW under the gates is likely to be caused by the difference in elasticity constants of the gates and substrate. In particular, the elasticity constants of the gates are greater that of the substrate. The gates are therefore much 'stiffer' than the substrate, and have a higher natu- (a)
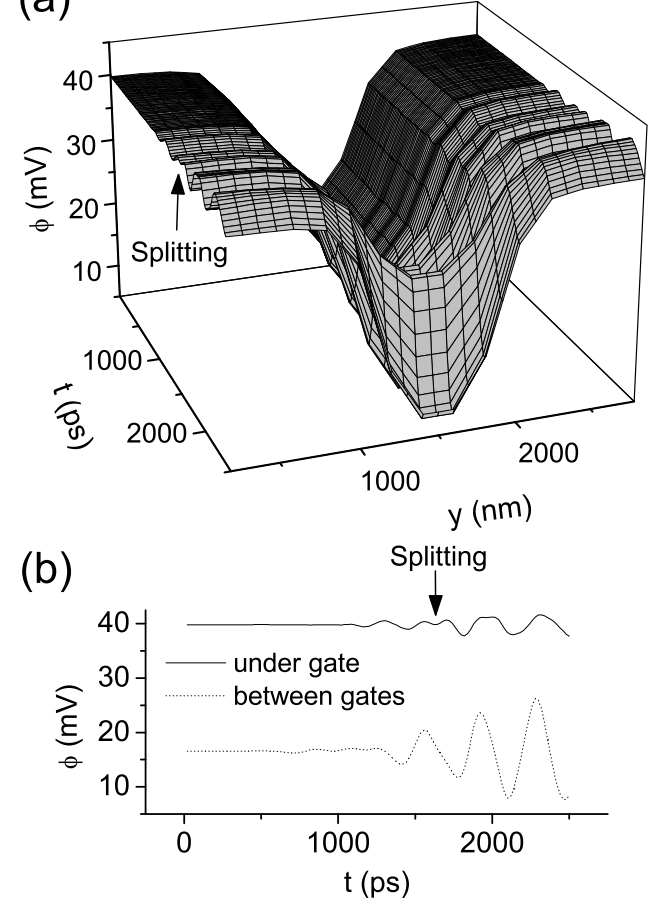

FIG. 8: (a) Time development of the total electric potential $\phi$ as a function of the $y$ coordinate. (b) The electric potential $\phi$ directly under the gate and in between the gate.

ral frequency of vibration. The vibration of surface gates impart additional vibrational components to the underlying SAW. A more detailed understanding of the mechanism behind the peak splitting would involve investigation of the lateral and vertical components of the displacements under a gate (and perhaps experiments with different gate materials), and therefore a major digression from the scope and intention of this paper but a possible topic of future work.

The simulated changes in amplitude and frequency of SAW would be undesirable for electron transport experiments as they may allow both tunnel and Coulomb interactions between electrons in consecutive SAW minima. However, these phenomena seem to be more significant for the first three peaks passing through the gate, than for the last peak. The last peak has a larger amplitude and thus greater energy, and is therefore more resistant to these effects.

Finally, from Fig. 8] one can see the absence of any significant turbulence in the SAW motion along the $y$ axis, which may have affected systems of onedimensional channels in parallel which are currently under development $\stackrel{29,30}{ }$ This also suggests that diffraction of the SAW on propagation though our split-gate is beyond the accuracy of our numerical work i.e. extremely small. Therefore the only waves in our system are plane waves which travel parallel to the lateral edges of the 
computational domain and are not reflected of them.

\section{CONCLUSION}

We have performed numerical simulations in order to investigate the dynamics resulting from the propagation of a piezoelectric SAW though a Q1DC defined by metallic split-gates on the surface of a $\mathrm{GaAs} / \mathrm{Al}_{X} \mathrm{Ga}_{1-X} \mathrm{As}$ heterostructure. Our simulations were performed in three spatial dimensions fully incorporating the mechanical and electrical parameters of the materials, and were based on the strongly screened, low SAW power and short constriction regime. We ignored the presence of static charges, as we are interested in the dynamical properties of the SAW as it propagates through a realistic split-gate device.

We have demonstrated significant 'amplitude reduction' of the SAW potential, up to $30 \%$ due to the mechanical presence of the surface gates, as well as up to $75 \%$ screening of the SAW potential, by the biased splitgate device. In addition, the simulations demonstrated the recovery of the SAW, after amplitude reduction due to the presence of mechanical gates, and also the absence of significant damping or screening effects at the center of the constriction formed by the split-gate. These effects have been demonstrated theoretically for the first time for a realistic device, and would be difficult, if possible at all, to achieve without a numerical procedure such as ours. The results suggest that the coherent propagation of SAWs through systems of Q1DCs in series or in parallel could also be achieved, although for definiteness, further simulations may be required.

Through the incorporation of a simple model for the screening by the 2DEG, we demonstrated a total electric potential modulation that resembled an electron pump and provided a simple model for the capture of electrons by the SAW, from the source $2 \mathrm{DEG}$, and the release of the electrons to the drain 2DEG.

\section{ACKNOWLEDGEMENTS}

We thank G. Gumbs, V. I. Talyanskii, A. M. Robinson, H. K. Bhadeshia, C. G. Smith, J. Jefferson and C. J. B Ford for comments and useful discussions. This work was partly funded by the UK EPSRC and QIP IRC. SR and MK acknowledge the Cambridge-MIT Institute for financial support.
* Electronic address: S.Rahman.00@cantab.net

${ }^{1}$ H. Matthews, Surface Wave Filters, John Wiley, 1st edition (1977).

2 P. Bierbaum, Appl. Phys. Lett. 21, 595 (1972).

3 A. Wixforth, J. P. Kotthaus, and G. Weimann, Phys. Rev. Lett. 56, 2104 (1986).

4 J. M. Shilton, V. I. Talyanskii, M. Pepper, D. A. Ritchie, J. E. F. Frost, C. J. B. Ford, C. G. Smith, and G. A. C. Jones, J. Phys.: Condens. Matter 8, L531 (1996).

5 C. H. W. Barnes, J. M. Shilton, and A. M. Robinson, Phys. Rev. B 62, 8410 (2000).

${ }^{6}$ G. R. Aizin, G. Gumbs, and M. Pepper, Phys. Rev. B 58, 589 (1998).

7 G. Gumbs, G. R. Aizin, and M. Pepper, Phys. Rev. B 57, 1654 (1998).

8 G. Gumbs, G. R. Aizin, and M. Pepper, Phys. Rev. B 60, 954 (1999).

9 S. Rahman, H. P. Langtangen and C. H. W. Barnes physics/0501075 (2005).

10 J. M. Shilton, D. R. Mace, V. I. Talyanskii, Y. Galperin, M. Y. Simmons, M. Pepper, and D. A. Ritchie, J. Phys. C 8, 337 (1996).

11 B. A. Auld, Acoustic Waves and Fields in Solids, vol. 1, Wiley (1973).

12 F. Alsina, P. V. Santos, H. P. Schönherr, R. Nötzel and K. H. Ploog, Phys. Rev. B 67, 161305(R), (2003).

13 T. Sogawa, P. V. Santos, S. K. Zhang, S. Eshlaghi, A. D. Wieck and K. H. Ploog, Phys. Rev. Lett. 87, 276601, (2003).

14 A. M. Robinson and C. H. W. Barnes, Phys. Rev. B 63, 165418 (2001).

15 P. A. Maksym, Phys. Rev. B 61, 4727 (2000).
16 K. Flensberg, Q. Niu, and M. Pustilnik, Phys. Rev. B 60, R16291 (1999).

17 S. E. Laux, D. J. Frank and F Stern, Surf. Sci. 196, (1988).

18 J. H. Davies, A. Larkin, and E. V. Sukhorukov, J. Appl. Phys. 77, 4504 (1995).

19 More realistic boundary conditions at the interface (between the substrate and any existing vacuum) may consist of either the continuity of Dz or charges $=0$, but the nature of the results presented here are such that they would not be affected by such changes to the boundary conditions.

20 To achieve this, we rotate the crystal by 45 degrees around the $z$ axis.

21 O. Zienkiewicz, The Finite Element Method, McGraw-Hill, 4th edition (1994).

22 H. P. Langtangen, Computational Partial Differential Equations - Numerical Methods and Diffpack Programming, Springer, 2nd edition (2003).

23 S. Adachi, Properties of Aluminium Gallium Arsenide, Institution of Electrical Engineers (1993).

${ }^{24}$ W. F. Gale and T. C. Tottmier, Smithells Metals Reference Book, Elsevier (2004).

25 S. H. Simon, Phys. Rev. B 54, 13878 (1996).

26 H. Pothier, P. Lafarge, C. Urbina, D. Esteve and M. H. Devoret, Europhys. Lett. 17, 249 (1992).

27 R. L. Kautz, M. W. Keller and J. M. Martinis, Phys. Rev. B 60, 8199 (1999).

28 J. Ebbecke, N. E. Fletcher, T. J. B. M. Janssen, F. J. Ahlers, M. Pepper, H. E. Beere and D. A. Ritchie, Phys. Lett. 84, 4319 (2004).

29 M. Kataoka, C. H. W. Barnes, C. J. B. Ford, H. E. Beere, D. A. Ritchie, D. Anderson, G. A. C. Jones and M. Pepper, to be published. 
${ }^{30}$ R. Rodriquez, D. K. L. Oi, M. Kataoka, C. H. W. Barnes, T. Ohshima and A. K. Ekert, Phys. Rev. B 72, 085329 (2005).

31 The strains produced by the voltages applied to the gates in this lower barrier regime, are small compared to those produced by the moving SAW and hence are not shown. 